\title{
Controlling Long-Lived Triplet Generation from Intramolecular Singlet Fission in the Solid State
}

Natalie A. Pace, Weimin Zhang, Dylan H. Arias, lain McCulloch, Garry Rumbles, and Justin C. Johnson J. Phys. Chem. Lett., Just Accepted Manuscript • DOI: 10.1021/acs.jpclett.7b02750 • Publication Date (Web): 30 Nov 2017

Downloaded from http://pubs.acs.org on December 5, 2017

\section{Just Accepted}

"Just Accepted" manuscripts have been peer-reviewed and accepted for publication. They are posted online prior to technical editing, formatting for publication and author proofing. The American Chemical Society provides "Just Accepted" as a free service to the research community to expedite the dissemination of scientific material as soon as possible after acceptance. "Just Accepted" manuscripts appear in full in PDF format accompanied by an HTML abstract. "Just Accepted" manuscripts have been fully peer reviewed, but should not be considered the official version of record. They are accessible to all readers and citable by the Digital Object Identifier (DOI®). "Just Accepted" is an optional service offered to authors. Therefore, the "Just Accepted" Web site may not include all articles that will be published in the journal. After a manuscript is technically edited and formatted, it will be removed from the "Just Accepted" Web site and published as an ASAP article. Note that technical editing may introduce minor changes to the manuscript text and/or graphics which could affect content, and all legal disclaimers and ethical guidelines that apply to the journal pertain. ACS cannot be held responsible for errors or consequences arising from the use of information contained in these "Just Accepted" manuscripts. 


\section{Controlling Long-Lived Triplet Generation from Intramolecular Singlet Fission in the Solid State}

Natalie A. Pace, ${ }^{1,2}$ Weimin Zhang, ${ }^{3}$ Dylan H. Arias, ${ }^{1}$ Iain McCulloch, ${ }^{3,4}$ Garry Rumbles, ${ }^{1,2,5}$ and Justin C. Johnson ${ }^{1 *}$

${ }^{1}$ National Renewable Energy Laboratory, 15013 Denver West Parkway, Golden, CO 80401, USA

${ }^{2}$ Department of Chemistry and Biochemistry, University of Colorado at Boulder, Boulder, CO, 80309, USA

${ }^{3}$ Center for Plastic Electronics, Imperial College London, London SW7 2AZ, United Kingdom

${ }^{4}$ KSC, Physical Sciences and Engineering Division, King Abdullah University of Science and Technology (KAUST), Thuwal 23955-6900, Saudi Arabia

${ }^{5}$ Renewable and Sustainable Energy Institute, University of Colorado at Boulder, Boulder, CO, 80309, USA

*Corresponding author email address: Justin.Johnson@nrel.gov 


\begin{abstract}
The conjugated polymer poly(benzothiophene dioxide) (PBTDO1) has recently been shown to exhibit efficient intramolecular singlet fission in solution. In this paper, we investigate the role of intermolecular interactions in triplet separation dynamics after singlet fission. We use transient absorption spectroscopy to determine the singlet fission rate and triplet yield in two polymers differing only by side chain motif in both solution and the solid state. Whereas solid-state films show singlet fission rates identical to those measured in solution, the average lifetime of the triplet population increases dramatically, and is strongly dependent on side-chain identity. These results show that it may be necessary to carefully engineer the solid-state microstructure of these "singlet fission polymers" in order to produce the long-lived triplets needed to realize efficient photovoltaic devices.
\end{abstract}

\title{
TOC Graphic:
}

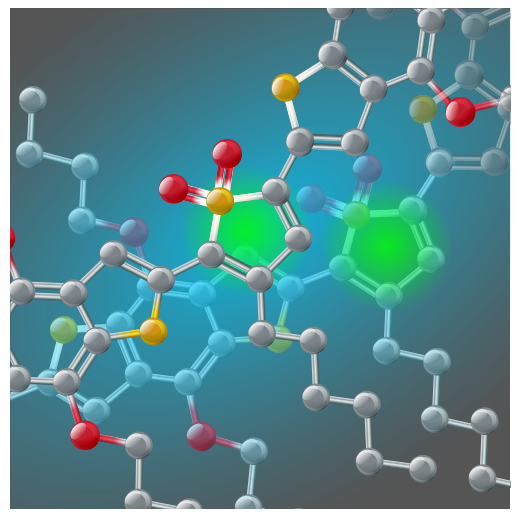


Singlet fission (SF) has the potential to increase solar cell efficiencies beyond the single-junction Shockley-Quiesser limit through the conversion of one singlet excited state $\left(\mathrm{S}_{1}\right)$ to two triplet excited states $\left(2 \mathrm{~T}_{1}\right)$, and subsequently two charge pairs per photon. ${ }^{1}$ While spin selection rules generally prohibit rapid conversion between singlets and triplets, the SF process can occur on an ultrafast timescale through a correlated triplet pair state $\left({ }^{1}(\mathrm{TT})\right)$, which conserves the spin of the $\mathrm{S}_{1}$ state. ${ }^{2}$ Both $\mathrm{SF}$ and triplet-driven electron transfer must be efficient in order to make a good solar cell that leverages SF for enhanced photocurrent. The first criterion has effectively been met, though the SF library needs to be expanded to address practical concerns (e.g. stability). The second criterion still presents a significant challenge. SF compounds like pentacene, tetracene, and 1,3diphenylisobenzofuran have been combined with solar cell mainstays like silicon, fullerenes, titanium dioxide, and semiconductor nanocrystals, but a complete understanding of triplet extraction and dissociation capable of surpassing $\sim 33 \%$ conversion efficiency has been elusive. ${ }^{2-7}$ Tuning triplet diffusion in these systems is limited because efficient intermolecular SF requires precise molecular orientations.

In contrast, intramolecular SF offers fine control over the SF rate through chemical bonds, with the tradeoff that many dimer and oligomer systems are limited by short triplet lifetimes. ${ }^{8-10}$ Intramolecular solutions to this problem may be limited, as a recent systematic study of pentacene oligomers showed no gain in triplet lifetime with the number of repeat units. ${ }^{11}$ However, it may be possible to extend triplet lifetimes through intermolecular interactions in the solid state, as was recently shown in films of tetracene oligomers. ${ }^{12}$ Polymer systems with extended conjugation lengths, tunable microstructure, and facile solution processing may be capable of striking a balance between SF rate, 
triplet separation efficiency, and practical concerns. Previously studied polymers have only exhibited limited SF from higher lying excited states, ${ }^{13-15}$ but poly(benzothiophene dioxide) (PBTDO1) is a new intramolecular SF system that forms triplets within $7 \mathrm{ps}$ at a $170 \%$ triplet yield. ${ }^{16}$ This system offers a unique opportunity to systematically examine the effect of intermolecular interactions on triplet dynamics. In this study, we examine two derivatives of PBTDO1 with linear and branched alkoxy side chains. Branched side chains have been shown to increase disorder in polymer films, while linear side chains tend to produce more ordered films with enhanced intermolecular coupling. ${ }^{17}$ We use transient absorption (TA) spectroscopy to study the dynamics of triplet production and decay in both solution and the solid state.

The two polymers used in this study are shown in Figure 1. Each has a different side chain on the benzodithiophene donor unit. The polymer with the linear side chains, or PBTDO1 "A", is the polymer that has previously exhibited fast triplet formation at $170 \%$ yield in solution. ${ }^{16}$ The polymer with the branched side chains, or PBTDO1 " $\mathrm{B}$ ", is a derivative synthesized here for the purpose of modifying the solid-state microstructure. Both polymers exhibit a red shift in their absorbance spectra of $\sim 100 \mathrm{meV}$ relative to solution when cast as solid films, with no significant change in spectral shape. 
(a)

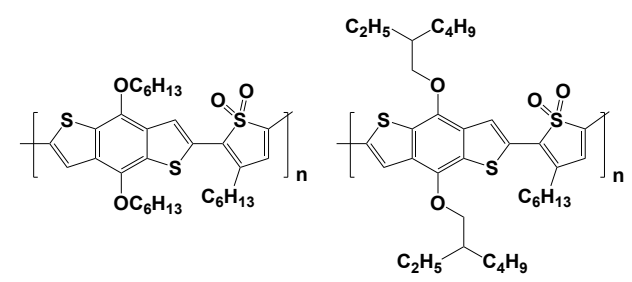

(b)

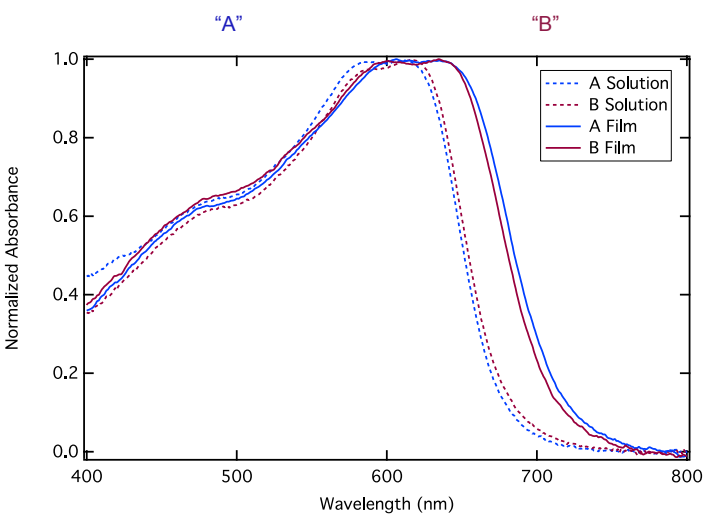

Figure 1: (a) Molecular structures for the repeat units of PBTDO1 “A” (left) and PBTDO1 " $B$ ” (right). (b) Absorption spectra for polymers $A$ and $B$ in chloroform solution and solid state.

Each spectro-temporal TA data set can be globally modeled with short, medium, and long decay times. The amplitude of each exponential varies as a function of wavelength, giving Decay Associated Spectra (DAS). The DAS for polymer A are shown in Figure 2. The DAS corresponding to the fast decay ( $\sim \mathrm{ps})$ of the initial singlet excitation are taken to represent the SF process. Both polymers A and B show evidence of SF in their TA dynamics in both the solution phase and the solid state. The TA spectra of $\mathrm{A}$ and $\mathrm{B}$ are essentially identical for both solutions and the solid state, and therefore only those of A are shown in Figure 2 (B dynamics in Figure S1). The time constant of SF for both polymers in chloroform, as well as both polymers cast as thin films from chloroform, is $\sim 4 \mathrm{ps}$, which is similar to the previously measured SF time of $7 \mathrm{ps}$ for $\mathrm{A}$ in chloroform. $^{16}$ 
(a)

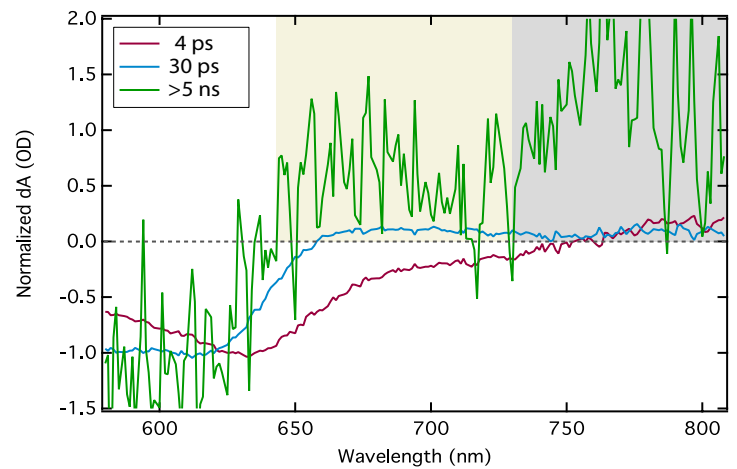

(b)

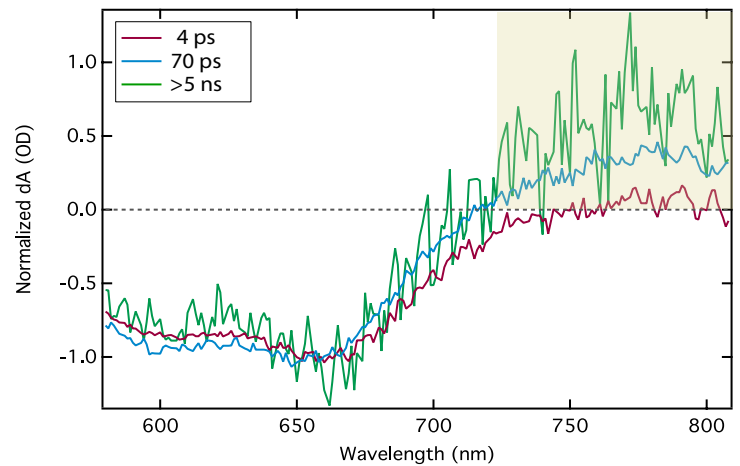

Figure 2: DAS from singular value decomposition (SVD) and global fit with three exponentials for polymer $A(a)$ in solution and (b) in the solid state. The time constants associated with each spectrum are $\sim 4$ ps (red spectra), 30-70 ps (blue spectra), and longer than $5 \mathrm{~ns}$ (green spectra). Triplet PIA spectral region is shaded in yellow, and singlet PIA is shaded in gray.

The DAS labeled 30 and 70 ps in Figure 2 correspond to the decay of initially formed triplets. Given the fast decay time, we specifically assign this spectral signal to coupled triplet pair states. The triplet spectral signature in the solid-state is verified through triplet sensitization with platinum (II) octaethyl porphyrin (Figure S3). The measured time of $\sim 30 \mathrm{ps}$ for triplet pair decay in solution is on the same order of magnitude as the previous measurement of $70 \mathrm{ps}$ for triplet decay. ${ }^{16}$ The systematically faster time constants measured in solution here cannot be explained by increased fluences, as all rates measured in solution are fluence-independent. The measured time constants for triplet pair decay increase to $\sim 70$ ps for the A thin film and $\sim 50$ ps for the B thin film at low fluence (Table 1). 


\begin{tabular}{|c|c|c|}
\hline & $\tau_{1}(\mathrm{ps})$ & $\tau_{2}(\mathrm{ps})$ \\
\hline A Solution & $3.2 \pm 0.7$ & $33 \pm 3$ \\
\hline B Solution & $4.4 \pm 0.7$ & $35 \pm 2$ \\
\hline A Film & $4 \pm 1$ & $69 \pm 8$ \\
\hline B Film & $4 \pm 0.5$ & $50 \pm 10$ \\
\hline
\end{tabular}

Table 1: Characteristic decay times from the DAS at low fluence. $\tau_{1}$ corresponds to the SF process and $\tau_{2}$ corresponds to triplet-triplet annihilation. The third characteristic decay time is $>5 \mathrm{~ns}$ for all samples.

The triplets that do not undergo initial $<100$ ps decay are represented by the spectra in Figure 2 for long times ( $>5 \mathrm{~ns})$, which are likely associated with separated triplet states. A species living longer than $1 \mathrm{~ns}$ in solution is also identified by Busby et al., and is assigned to a charge-transfer state. However, the spectral differentiation between the long-lived state and the short-lived triplet state in solution could be the result of the larger relative contribution of singlet photoinduced absorption (PIA) at long times (Fig. 2a, gray shaded area). This is consistent with the observation of delayed fluorescence from triplet pair state recombination, ${ }^{16}$ which would repopulate the singlet state on a timescale of tens of picoseconds. Since triplet PIA is still visible in the $>5 \mathrm{~ns}$ time component in solution, this component is assigned to a mixture between a long-lived triplet state and a singlet state re-formed from fusion of the coupled triplet pair state. The $>5$ ns time component in the solid state matches the short-lived triplet spectrum more closely, with no evidence of singlet PIA. The lifetime of this state was measured to be as long as $200 \mathrm{~ns}$ in the A film (Figure S4). 


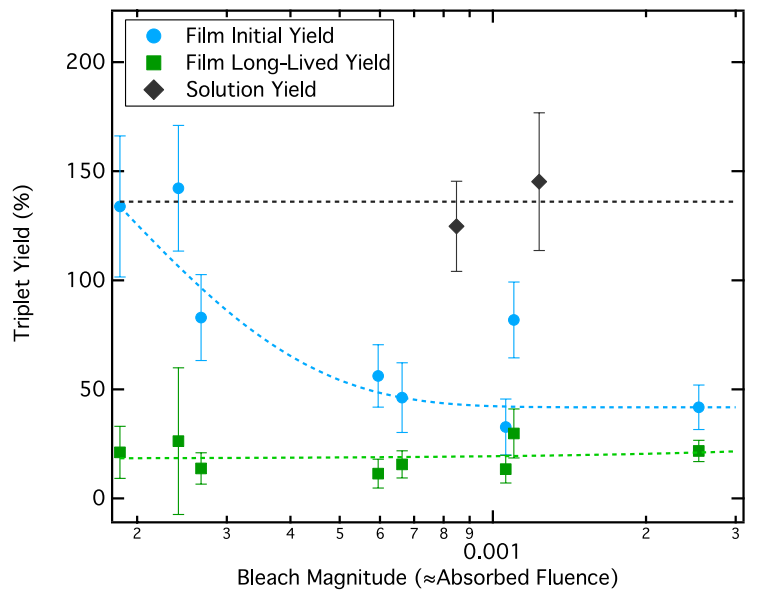

Figure 3: Triplet yields as a function of bleach magnitude for the A thin film.

PBTDO1 A and tetracene solutions were excited under identical conditions, and the PBTDO1 A triplet yield $\Phi_{\mathrm{T}}$ was determined using a measured polymer triplet extinction coefficient (see SI for details) and literature values for the tetracene extinction coefficient and tetracene intersystem crossing yield: ${ }^{18}$

$$
\Phi_{T}=\Phi_{I S C} \frac{\Delta A_{P B T D O 1}}{\varepsilon(T)_{P B T D O 1}} \frac{\varepsilon(T)_{\text {Tetracene }}}{\Delta A_{\text {Tetracene }}}
$$

The triplet yields for A and B solutions are $130 \pm 20 \%$ and $150 \pm 30 \%$, respectively. The triplet yields for the films decrease as a function of fluence, but at low fluence converge to the yield, similar to that of the solution measurements, of $\sim 140 \%$ (Figure 3 ). The decrease in yield with increasing fluence is likely due to increased singlet-singlet annihilation (SSA), as the time component associated with singlet decay also decreases as function of fluence. SSA can become a predominant pathway for singlet decay in polymer thin films at laser fluences of $\sim 10^{13}$ photons $/ \mathrm{cm}^{2} 19,20$ as a result of faster interchain exciton hopping compared to intrachain exciton hopping. ${ }^{21}$ Our measurements range from $10^{12}$ to $10^{14} \mathrm{~cm}^{2}$. Although our lowest fluence measurements converge with 
solution triplet yields, our measurements at higher fluence fall into a range where SSA can dominate the dynamics and limit $\Phi_{\mathrm{T}}$.

\begin{tabular}{|c|c|}
\hline & $\Phi_{\text {T long }}(\%)$ \\
\hline A Solution & $4 \pm 1$ \\
\hline B Solution & $4 \pm 1$ \\
\hline A Film & $24 \pm 4$ \\
\hline B Film & $12 \pm 1$ \\
\hline
\end{tabular}

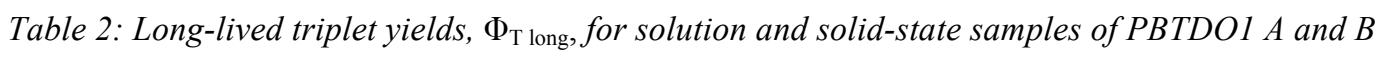

In contrast, the long-lived triplet yield, $\Phi_{\mathrm{T} \text { long, }}$, has no fluence dependence. Significant differences arise between the films and solutions, as well as between the A and B films, in the behavior of the long-lived triplet. In Table 2, we compare $\Phi_{\mathrm{T}}$ long for the four samples. Both A and B solutions have the same small $\Phi_{\mathrm{T} \text { long. }}$ Both A and B films have substantially higher long-lived triplet yields than the solutions. However, whereas the B film produces only a three-fold increase in $\Phi_{\mathrm{T} \text { long }}$ relative to solution, the A film produces a six-fold increase.

We performed a series of temperature-dependent, steady-state and TA measurements on the A polymer solution to learn more about the source of the increased long-lived triplet population in the A film. Chlorobenzene is used in these experiments to facilitate a larger range of available solvent temperatures than chloroform. At room temperature, the A absorption spectrum in chlorobenzene is almost identical to the absorption spectrum in chloroform (Figure S6). Further details on the subtle differences in dynamics between the two solvents can be found in the SI (Figure S7). 
(a)

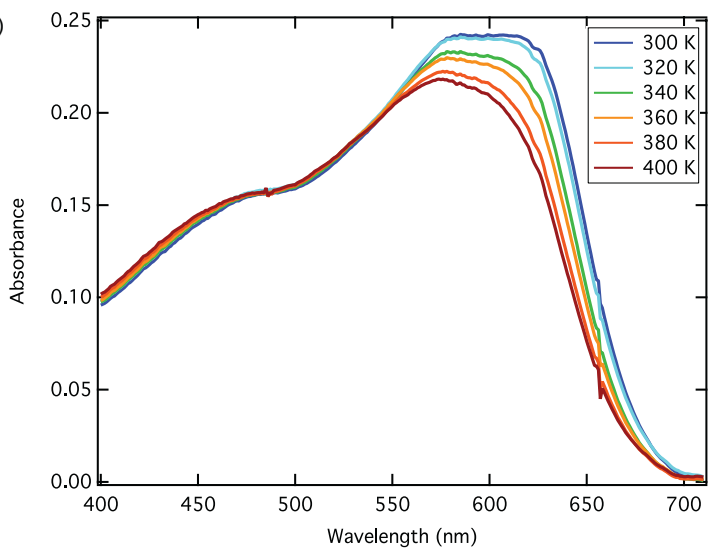

(b)

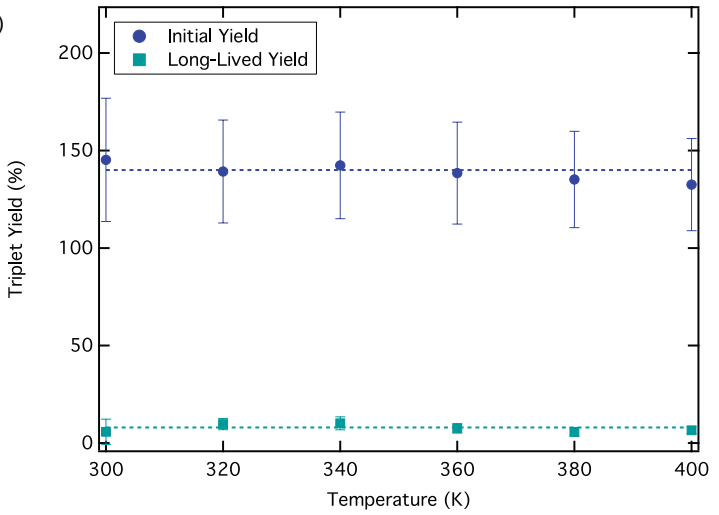

Figure 4: (a) Steady-state absorption spectra of the A solution at a series of temperatures from $300 \mathrm{~K}$ to $400 K$. (b) Initial and long-lived triplet yields of the A solution as a function of temperature.

As shown in Figure 4a, the steady-state absorption spectrum of the PBTDO1 A/chlorobenzene solution changes as a function of temperature, with decreasing intensity on the red edge of the spectrum as the temperature increases. This type of thermochromism is likely intramolecular in nature, where increased temperatures activate new torsional modes and decrease the average conjugation length. Identical absorption behavior as a function of temperature was observed in polydiacetylenes, where this transition was specifically assigned to a single-chain rigid rod to coil conformational change. ${ }^{22,23}$ Interestingly, these conformational changes do not result in a change in the initial triplet yield or long-lived triplet yield (Figure 4b). The average initial triplet yield is $140 \pm 10 \%$ and the average long-lived triplet yield is $8 \pm 2 \%$ across the entire 
temperature range. We thus find that the classifications of SF as intramolecular and nonactivated in these systems is likely correct. The invariance of the SF rate with phase or side-chain identity further supports the intramolecular mechanism.

All PBTDO1 samples produce two distinct populations of triplets. The first population decays within 30-70 ps, and the second population lives longer than $5 \mathrm{~ns}$. Both populations are formed by SF, as the triplet signal rises with a single time constant of a few picoseconds. While all the TA data presented is fit with a tri-exponential function, fits to the data using a branched kinetic model can be found in Figure S8. A sequential model fits the data equally well, but a branched model is more likely because the two triplet populations have different fluence dependences. Given the difference in decay times of several orders of magnitude, the first population is assigned to triplet pair states confined to the same polymer chain, and the second population is assigned to separated triplet states on different polymer chains. The two branches of the kinetic scheme are shown in Figure 5. The yield of separated triplets is determined by the ratio between favorable and unfavorable triplet separation sites. 
(a)

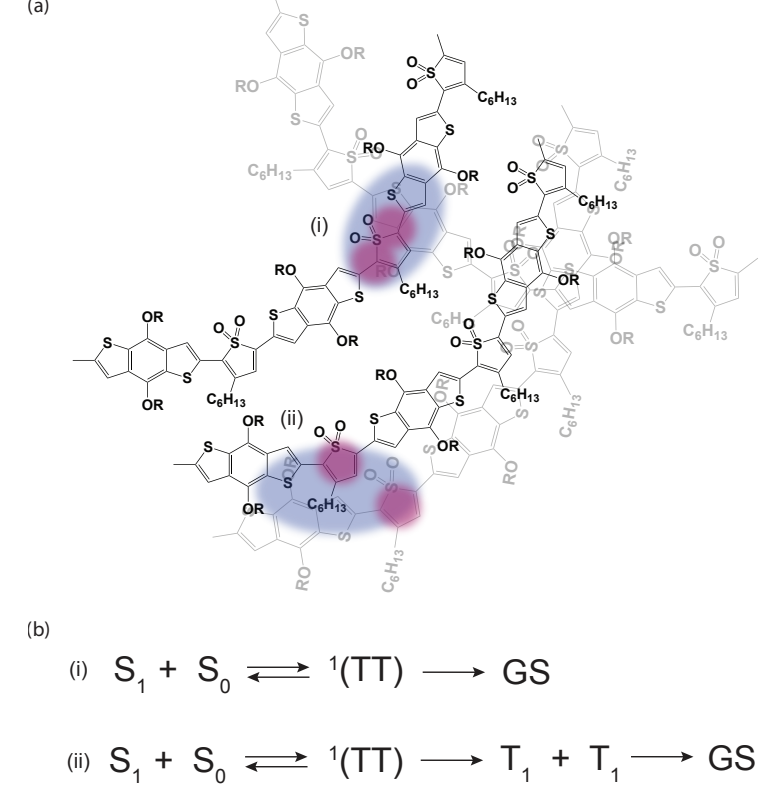

Figure 5: (a) Cartoon and (b) Kinetic scheme that show two different types of SF sites in a polymer thin film, where case (i) is a site unfavorable to free triplet formation and case (ii) is a site favorable to free triplet formation. The purple species denote delocalized singlet excitations, while the red species denote a coupled triplet pair in case (i) and separated triplets in case (ii). The relative orientations of the polymer chains at these sites are shown for illustrative purposes, and were not directly measured in this study.

In solution, both the initial and long-lived triplet yields are insensitive to fluence. Therefore, these yields represent intrinsic initial and long-lived triplet yields. The presence of a long-lived triplet species in solution is likely a consequence of some small degree of intrachain triplet separation from sufficiently delocalized singlet excitations or certain favorable chain conformations. Since the long-lived triplet yields are relatively low, it is difficult to distinguish differences in long-lived triplet yield between the A and B solutions.

The rates of SF, triplet-triplet annihilation, and the decay of separated triplets remain relatively constant between the solution and film. However, the concentrations of all the species involved in these processes increases due to the large density of polymer chains in the films compared with solutions. As a result, in films we observe a decrease in the singlet decay time component and a decrease in the initial triplet yield as a function of 
fluence from SSA. In contrast, the long-lived triplet yield is constant with fluence, which we attribute to a slower rate of SSA at sites of efficient triplet separation. SSA rates have been shown to decrease by an order of magnitude in poly(3-hexylthiophene) (P3HT) films as a function of molecular structure, where slower rate constants are associated with more red-shifted emission. ${ }^{24}$ We expect that in PBTDO1, similar variation in SSA rates as function of microstructure could occur. This would lead to regions with slower SSA that even at high fluence provide little competition for the fluence- and microstructureindependent SF. These regions may simultaneously represent sites for efficient triplet separation, thus producing the fluence-independent long-lived triplet yield. In other words, there may be low-energy portions of the PBTDO1 microstructure optimal for long-lived triplet generation, but sub-optimal for SSA. In this context, our findings underscore the importance of controlling conditions for efficient triplet separation in future work.

Both films produce a significantly increased $\Phi_{\mathrm{T} \text { long }}$ compared to the solutions. This result suggests that the high density of polymer chains in a thin film environment specifically facilitate triplet separation through interchain interactions. Furthermore, the A film produces twice as many long-lived triplets as the B film. Thus, solid-state microstructure is key to optimizing triplet separation efficiency in polymer thin films. Future work will focus on optimizing interchain interactions through chemical design, as shown in this study, as well as parameters like solution processing conditions. A wider variety of thin film environments across a range of polymeric systems is needed in order to develop more specific guidelines for facilitating triplet extraction via interchain interactions. 


\section{Experimental Methods:}

Synthesis: Both polymers were synthesized using palladium catalyzed Suzuki coupling reactions. Further details can be found in the SI.

Sample Fabrication: Both polymers were dissolved in chloroform at an approximate optical density of 0.1 ( $2 \mathrm{~mm}$ pathlength). Before TA, nitrogen was used to purge both samples for 15 minutes. For temperature-dependent TA, the same general preparation was used with chlorobenzene as the solvent. Both films were blade coated on quartz substrates from $7.5 \mathrm{mg} / \mathrm{mL}$ solutions of PBTDO1 A or B in chloroform. Before TA, both films were sealed using Surlyn and a glass slide heated at $\sim 95^{\circ} \mathrm{C}$ in a nitrogen atmosphere.

Absorption Measurements: All steady state absorption measurements were taken using a UV-Vis Shimadzu spectrometer. An integrating sphere was used for the film measurements. TA experiments were conducted using a Coherent Libra Ti:sapphire laser with a power output of $\sim 4 \mathrm{~W}$, a repetition rate of $1 \mathrm{kHz}$, and a pulse width of $\sim 100 \mathrm{fs}$ at $800 \mathrm{~nm}$. Approximately thirty percent of the total output was apportioned to generate 550 nm excitation light using an TOPAS commercial optical parametric amplifier. A portion of the $800 \mathrm{~nm}$ output was also used to generate a broadband "probe" beam using a sapphire crystal or calcium fluoride crystal. The broadband beam was split into two beams in order to more accurately track absorptive changes with a reference spectrum. An optical chopper was used to reduce the excitation light repetition rate to $500 \mathrm{~Hz}$ so that only alternating probe pulses coincided with pump pulses. Unless otherwise specified, delay times were controlled using a multipass delay stage capable of creating $\sim 5$ ns of delay time between the pump and probe pulses. When a longer time window was 
required, the delay time was manipulated electronically using an electro-optical system (EOS). Since this detection system has a longer time resolution, it was only used to determine the lifetime of the long-lived triplet. The pump beam was adjusted to maximally overlap the probe beam at the sample position. Photon fluences were determined using a series of pinholes between 25 and $250 \mu \mathrm{m}$ assuming a Gaussian density distribution. A Janis VPF 100 Cryostat was used for the temperature-dependent TA measurements. All data was recorded and analyzed using Ultrafast Systems' Surface Explorer Software. All signals were quantified using singular value decomposition and global fitting, which does not specify a particular kinetic model.

\begin{abstract}
N.A.P, D.H.A, G.R., and J.C.J. acknowledge the Solar Photochemistry Program of the U.S. Department of Energy, Office of Basic Energy Sciences, Division of Chemical Sciences, Biosciences, and Geosciences under contract DE-AC36-08GO28308 with NREL for support of spectroscopy, data analysis, and sample preparation. W.Z. and I.M. thank EPSRC Project EP/M005143/1 and EC FP7 Project SC2 (610115) for support of the polymer synthesis, purification, and characterization. We thank Obadiah Reid and Wade Braunecker for useful discussions.
\end{abstract}

\footnotetext{
Supporting Information. TA data for PBTDO1 B, TA data for all samples without normalization, triplet sensitization, EOS TA data, triplet yield determination, solvent dependence, kinetic modeling, synthesis details
} 
1. Hanna, M. C.; Nozik, A. J. Solar Conversion Efficiency of Photovoltaic and Photoelectrolysis Cells with Carrier Multiplication Absorbers. J. Appl. Phys. 2006, $100,074510$.

2. Smith, M. B.; Michl, J. Singlet Fission. Chem. Rev. 2010, 110, 6891-6936.

3. Tritsch, J. R.; Chan, W.-L.; Wu, X.; Monahan, N. R.; Zhu, X. Y. Harvesting Singlet Fission for Solar Energy Conversion via Triplet Energy Transfer. Nat. Commun. 2013, 4, 1-7.

4. Schrauben, J. N.; Zhao, Y.; Mercado, C.; Dron, P. I.; Ryerson, J. L.; Michl, J.; Zhu, K.; Johnson, J. C. Photocurrent Enhanced by Singlet Fission in a DyeSensitized Solar Cell. ACS Appl. Mater. Interfaces 2015, 7, 2286-2293.

5. Monahan, N.; Zhu, X. Y. Charge Transfer-Mediated Singlet Fission. Annu. Rev. Phys. Chem. 2015, 66, 601-618.

6. Thompson, N. J.; Wilson, M. W. B.; Congreve, D. N.; Brown, P. R.; Scherer, J. M.; Bischof, T. S.; Wu, M.; Geva, N.; Welborn, M.; Voorhis, T. V.; et al. Energy Harvesting of Non-Emissive Triplet Excitons in Tetracene by Emissive $\mathrm{PbS}$ Nanocrystals. Nat. Mater. 2016, 13, 1039-1043.

7. Lee, J.; Jadhav, P.; Reusswig, P. D.; Yost, S. R.; Thompson, N. J.; Congreve, D. N.; Hontz, E.; Van Voorhis, T.; Baldo, M. A. Singlet Exciton Fission Photovoltaics. Acc. Chem. Res. 2013, 46, 1300-1311.

8. Zirzlmeier, J.; Lehnherr, D.; Coto, P. B.; Chernick, E. T.; Casillas, R.; Basel, B. S.; Thoss, M.; Tykwinski, R. R.; Guldi, D. M. Singlet Fission in Pentacene Dimers. Proc. Natl. Acad. Sci. U.S.A. 2015, 112, 5325-5330.

9. Petelenz, P.; Snamina, M.; Mazur, G. Charge-Transfer States in Pentacene: Dimer Versus Crystal. J. Phys. Chem. C 2015, 14338-14342.

10. Vallett, P. J.; Snyder, J. L.; Damrauer, N. H. Tunable Electronic Coupling and Driving Force in Structurally Well-Defined Tetracene Dimers for Molecular Singlet Fission: a Computational Exploration Using Density Functional Theory. $J$. Phys. Chem. A 2013, 117, 10824-10838.

11. Sanders, S. N.; Kumarasamy, E.; Pun, A. B.; Steigerwald, M. L.; Sfeir, M. Y.; Campos, L. M. Singlet Fission in Polypentacene. Chem 2016, 1, 505-511.

12. Pun, A. B.; Sanders, S. N.; Kumarasamy, E.; Sfeir, M. Y.; Congreve, D. N.; Campos, L. M. Triplet Harvesting From Intramolecular Singlet Fission in Polytetracene. Adv. Mater. 2017, 258, 1701416.

13. Zhai, Y.; Sheng, C.; Vardeny, Z. V. Singlet Fission of Hot Excitons in Conjugated Polymers. Phil. Trans. R. Soc. A. 2015, 373, 20140327.

14. Guo, J.; Ohkita, H.; Benten, H.; Ito, S. Near-IR Femtosecond Transient Absorption Spectroscopy of Ultrafast Polaron and Triplet Exciton Formation in Polythiophene Films with Different Regioregularities. J. Am. Chem. Soc. 2009, 131, 1686916880.

15. Tamai, Y.; Ohkita, H.; Benten, H.; Ito, S. Singlet Fission in Poly(9,9'-Di- NOctylfluorene) Films. J. Phys. Chem. C 2013, 117, 10277-10284.

16. Busby, E.; Xia, J.; Wu, Q.; Low, J. Z.; Song, R.; Miller, J. R.; Zhu, X. Y.; Campos, L. M.; Sfeir, M. Y. A Design Strategy for Intramolecular Singlet Fission Mediated by Charge-Transfer States in Donor-Acceptor Organic Materials. Nat. Mater. 2015, 14, 426-433.

17. Fall, S.; Biniek, L.; Odarchenko, Y.; Anokhin, D. V.; de Tournadre, G.; Leveque, 
von, P.; Leclerc, N.; Ivanov, D. A.; Simonetti, O.; Giraudet, L.; et al. Tailoring the Microstructure and Charge Transport in Conjugated Polymers by Alkyl Side-chain Engineering. J. Mater. Chem. C 2016, 4, 286-294.

18. Burgdorff, C.; Ehrhardt, S.; Löhmannsröben, H. G. Photophysical Properties of Tetracene Derivatives in Solution. J. Phys. Chem. 2001, 95, 4246-4249.

19. Yu, J.; Lammi, R.; Gesquiere, A. J.; Barbara, P. F. Singlet-Triplet and Triplet-Triplet Interactions in Conjugated Polymer Single Molecules. J. Phys. Chem. B 2005, 109, 10025-10034.

20. Zaushitsyn, Y.; Jespersen, K. G.; Valkunas, L.; Sundström, V.; Yartsev, A. Ultrafast Dynamics of Singlet-Singlet and Singlet-Triplet Exciton Annihilation in Poly(3- 2'-Methoxy- 5'Octylphenyl)Thiophene Films. Phys. Rev. B 2007, 75, 223.

21. Tamai, Y.; Ohkita, H.; Benten, H.; Ito, S. Exciton Diffusion in Conjugated Polymers: From Fundamental Understanding to Improvement in Photovoltaic Conversion Efficiency. J. Phys. Chem. Lett. 2015, 6, 3417-3428.

22. Rughooputh, S.; Hotta, S.; Heeger, A. J. Chromism of Soluble Polythienylenes. J. Polym. Sci. Part B: Polym. Phys. 1987, 25, 1071-1078.

23. Lim, K. C.; Heeger, A. J. Spectroscopic and Light Scattering Studies of the Conformational (Rod-to-Coil) Transition of Poly(Diacetylene) in Solution. $J$. Chem. Phys. 1985, 82, 522-530.

24. Cook, S.; Liyuan, H.; Furube, A.; Katoh, R. Singlet Annihilation in Films of Regioregular Poly(3-Hexylthiophene): Estimates for Singlet Diffusion Lengths and the Correlation Between Singlet Annihilation Rates and Spectral Relaxation. $J$. Phys. Chem. C 2010, 114, 10962-10968. 